\title{
Cellular Localization of the MPP4 Protein in the Mammalian Retina
}

\author{
Heidi Stöbr, Jelena Stojic, and Bernhard H. F. Weber
}

Purpose. Membrane protein, palmitoylated (MPP)- 4 is a novel retina-specific member of the p55-like subfamily of membraneassociated guanylate kinases (MAGUKs). MAGUKs are known to act as scaffolding molecules for multiprotein complexes at specialized regions of the plasma membrane. The goal of this study was to characterize the MPP4 protein and to determine its location in the mammalian retina.

Methods. RT-PCR and 5' and $3^{\prime}$ rapid amplification of cDNA ends (RACE) techniques were used to isolate and sequence the full-length bovine MPP 4 cDNA. Polyclonal antisera against the bovine MPP4 protein were generated in rabbits immunized with synthetic peptides. Affinity-purified anti-MPP4 antibodies were used to investigate the properties and distribution of MPP4 in retina and transfected 293-Ebna cells by Western blot analysis and immunofluorescence microscopy.

Results. The full-length bovine MPP 4 cDNA encodes a putative bovine MPP4 protein of 640 amino acids with a predicted molecular mass of $72.9 \mathrm{kDa}$. Affinity-purified anti-MPP4 antibodies specifically detected the MPP4 protein in extracts from retina and 293-Ebna cells transfected with the MPP4 cDNA. Immunofluorescence analyses revealed that both the bovine and porcine MPP 4 proteins are localized in the connecting cilia and synaptic terminals of cone and rod photoreceptors. In addition, postsynaptic structures in the outer plexiform layer and three distinct bands in the inner plexiform layer were MPP4-positive. In porcine but not bovine retina, a subclass of cone bipolar cells were labeled with anti-MPP4.

Conclusions. The concurrent presence of MPP 4 in connecting cilia and synaptic structures suggests that MPP4 plays a role at membrane-cytoskeleton interfaces in distinct structural and functional compartments of bovine and porcine retinas. This work provides the basis for further investigations into the function of MPP4 as a retinal scaffolding molecule and its possible role in retinal disease. (Invest Ophthalmol Vis Sci. 2003;44:5067-5074) DOI:10.1167/iovs.03-0702

M embrane-associated guanylate kinases (MAGUKs) are proteins found in organisms from many phyla and are typically organized in a tripartite domain structure consisting of one to three PDZ domains, an $s r c$ homology 3 (SH3) motif, and a guanylate kinase-like (GUK) region. ${ }^{1}$ Additional conserved motifs present in some but not all MAGUKs include the L27 heterodimerization domain, the caspase recruitment domain, a

From the Institute of Human Genetics, Biocentre, University of Würzburg, Würzburg, Germany.

Supported by German Research Foundation (DFG) Grant STO 366/2-1 and by Federal Ministry of Education and Research (BMBF) Grant 01KW9921.

Submitted for publication July 8, 2003; revised August 19, 1003; accepted August 28, 2003.

Disclosure: H. Stöhr, None; J. Stojic, None; B.H.F. Weber, None

The publication costs of this article were defrayed in part by page charge payment. This article must therefore be marked "advertisement" in accordance with 18 U.S.C. $\$ 1734$ solely to indicate this fact.

Corresponding author: Heidi Stöhr, Institut für Humangenetik, Biozentrum, Am Hubland, D-97074 Würzburg, Germany;

heidi.stoehr@mail.uni-wuerzburg.de.
$\mathrm{Ca}^{2+} /$ calmodulin-dependent protein kinase sequence, and HOOK regions containing a binding site for the cytoskeletal protein $4.1{ }^{1-3}$ The various protein modules share the capability of mediating intra- and/or intermolecular interactions, thereby enabling the MAGUKs to act as scaffolding proteins for large multiprotein complexes. At the cellular level, MAGUK proteins are localized to specialized compartments underlying the plasma membrane at sites of cell-cell contacts such as synapses or tight junctions, where they bind and cluster transmembrane receptors and ion channels, concatenate cytoskeletal elements, and organize large signaling networks. ${ }^{1}$

Thus far, studies on MAGUK proteins in the mammalian retina have mainly been concentrated on the neuronal synapseassociated proteins (SAPs) that belong to the structurally related Drosophila discs large tumor suppressor gene (Dlg)-like MAGUK subfamily. These include PSD-95 (SAP90), PSD-93 (chapsin-110), SAP102, and SAP97, proteins that are known to be essential for synaptic function and morphology by anchoring ion channels, neurotransmitter receptors, and cell adherens molecules. ${ }^{1}$ In the rat retina, immunocytochemical analyses showed that the four proteins are exclusively expressed at the synaptic sites of the outer (OPL) and inner (IPL) plexiform layers, where they display a clustered distribution. Labeling of PSD-95, PSD-93, and SAP97 in the OPL was presynaptic and was restricted to the inner synaptic membranes of the photoreceptor terminals. ${ }^{4,5}$ During ontogenesis, these SAPs show a differential distribution, indicating a specific role of each member in the establishment of synaptic contacts. ${ }^{5}$ SAP102 is a postsynaptic protein in the OPL and has been localized to invaginating axonal processes of horizontal cells. ${ }^{6}$ In the IPL, all four SAPs presumably colocalize at postsynaptic sites. Electron micrographs of PSD-95 or SAP102 labeling indicated an expression postsynaptic to bipolar cell ribbon synapses in the IPL; however, only one of the two processes at the bipolar cell dyad, amacrine or ganglion cell dendrites, was immunopositive. ${ }^{4,6}$

MAGUK proteins of the p55 subfamily are increasingly recognized as playing an important role in retinal structure and function. The $\mathrm{p} 55$ family constitute a subclass of MAGUKs exemplified by the major palmitoylated erythrocyte membrane protein MPP1 and currently consists of six members (MPP1 to -6) identified in various species. p55-like proteins have been demonstrated to bind to a variety of protein partners including other MAGUKs. Most interesting, MPP5 (PALS1) has recently been reported to bind the mammalian homologue of Drosophila Crumbs, CRB1, ${ }^{7}$ a photoreceptor-specific protein that, when mutated, causes severe forms of retinitis pigmentosa (RP12) and Leber congenital amaurosis. ${ }^{8,9}$ In addition to CRB1, MPP5 also interacts with the multiple PDZ domain protein PATJ (PALS1-associated tight junction). In cultured epithelial cells, the assembled complex localizes to tight junctions. ${ }^{7}$ Similarly, the orthologous Drosophila proteins Crumbs, Stardust (orthologue to mammalian MPP5), and Discs Lost (orthologue to mammalian PATJ) form a complex at the marginal zone region of the zonula adherens. ${ }^{10-12}$ Both vertebrate and invertebrate protein complexes have been implicated in the organization of cell junctions and the regulation of epithelial polarity. More recently, it has been shown that Drosophila 
Crumbs also plays a role in photoreceptor morphogenesis. Crumbs maintains the zonula adherens integrity that facilitates stalk extension and rhabdomere elongation. ${ }^{13,14}$ The localization of Drosophila Crumbs and mouse Crb1 to functionally equivalent apical membrane regions of photoreceptors, the stalk of Drosophila rhabdomeres, and the inner segments (IS) of mouse rod and cone photoreceptors, strongly suggests a similar function of the mammalian counterparts. ${ }^{13}$ Although the precise role of Stardust or the mammalian MPP5 proteins in retinal physiology is not known, evidence for its involvement in retinal development comes from the analysis of zebrafish mutants displaying a loss of retinal architecture. Larval-lethal mutations in the nagie oko (nok) gene encoding the zebrafish orthologue of MPP5 produce severe disorganization of the retina with a lack of proper polarity of neuroepithelial sheets. ${ }^{15}$ Similar to Crumbs in Drosophila and Crb1 in mouse, nok localizes to apical membranes of photoreceptors and is concentrated immediately apical to the zonula adherens, also known as the outer limiting membrane (OLM).

Recently, we have isolated and characterized the human gene encoding the p55-like MAGUK protein MPP4. ${ }^{16}$ Expression studies using reverse transcriptase (RT)-PCR and Northern blot analyses have demonstrated that MPP4 is abundantly transcribed in the human retina, implying yet another MAGUK family member in the cellular processes underlying vision. As a first step toward elucidation of MPP 4 function, we cloned the bovine MPP 4 cDNA and generated specific peptide antibodies. These were used to determine the cellular distribution of MPP4 in the bovine and porcine retina. Herein, we present data showing that MPP 4 is expressed in the connecting cilia and the synaptic terminals of cone and rod photoreceptors. In addition, postsynaptic elements in the OPL, as well as three bands with varicose structures in the IPL, were labeled with the anti-MPP 4 antibody. In pig, MPP 4 was found in a subset of cone bipolar cell bodies. Extraction studies performed with retinal tissue indicate that MPP4 is associated with membrane-bound cytoskeletal structures in the retinal cells.

\section{Materials and Methods}

\section{Isolation of Bovine MPP4 CDNA}

Total RNA was extracted from bovine retina and reverse transcribed as described elsewhere. ${ }^{16}$ The full-length bovine MPP4 cDNA was identified by a combination of RT-PCR with primers designed from the human MPP4 cDNA sequence (GenBank, AF316032; http://www. ncbi.nlm.nih.gov/Genbank; provided in the public domain by the National Center for Biotechnology Information, Bethesda, MD), and 5' and $3^{\prime}$ rapid amplification of cDNA ends (RACE). Primer pair bovF 4 (5'-CGG CAA GGA GGA TGA TAC AGT-3') and bovR8 (5'-CCA GTA TTA AGC AGA AGG C-3') was used in RT-PCR to amplify the complete coding region from bovine retinal RNA and to confirm the cDNA sequence.

\section{Transient Expression in Ebna-293 Cells}

For heterologous expression experiments, the full-length bovine MPP4 cDNA was inserted into the pCEP4 vector (Invitrogen, Carlsbad, CA). Ebna-293 cells (Invitrogen) were maintained in DMEM supplemented with $10 \%$ FCS, $2 \mathrm{mM}$ L-glutamine, and $250 \mu \mathrm{g} / \mathrm{mL} \mathrm{G} 418$. Transfection was performed with $20 \mu \mathrm{g}$ of expression plasmid per $10-\mathrm{cm}$ tissue culture plate, using the standard calcium phosphate method. Cells were harvested after 2 days and solubilized in PBS and 1\% Triton X-100 for 30 minutes at $4^{\circ} \mathrm{C}$. After centrifugation at $16,000 \mathrm{~g}$ for 30 minutes, the supernatant was retained for SDS-polyacrylamide gel electrophoresis (SDS-PAGE). For immunofluorescence microscopy cells were grown on laminin-coated coverslips, fixed in $4 \%$ paraformaldehyde for 30 minutes, and immunolabeled.

\section{Antibodies}

Anti-MPP4 antisera were generated by immunizing rabbits with the bovine peptide sequence $\mathrm{NH}_{2}$-IQSDKGSKLPDEKDKC-CONH$H_{2}$ coupled to keyhole limpet hemocyanin (Eurogentec, Seraing, Belgium). The polyclonal antibodies were affinity-purified with the MPP4 peptide conjugated to an EAH-Sepharose column. The affinity-purified antibody was used at a 1:1000 dilution for Western blot analysis and a 1:400 dilution for immunofluorescence.

Other antibodies used for immunofluorescence included mouse monoclonal anti-PSD-95 clone 7E3-1B8 (1:200; Affinity Bioreagents, Golden, CO), anti-VGLUT1 clone F123 (1:500; Synaptic Systems, Göttingen, Germany), anti-calbindin D-28K clone CB-955 (1:500; SigmaAldrich, St. Louis, MO), anti-calretinin clone CRT01 (1:50; NeoMarkers, Fremont, CA), anti-PKC $\alpha$ clone MC5 (1:200; Sigma-Aldrich), rat olfactory bulb (ROB) clone 115A10 (1:2000; kindly provided by Shinobu C. Fujita, Mitsubishi Kasei Institute of Life Sciences, Tokyo, Japan), antiglutamine synthetase (1:1000; kindly provided by Andreas Reichenbach, University of Leipzig, Leipzig, Germany), anti-rhodopsin clone $1 \mathrm{D} 4^{17}(1: 40)$ and anti-RS1 clone $3 \mathrm{R}^{10} 0^{18}$ (1:50). The latter two clones were kindly provided by Robert S. Molday (University of British Columbia, Vancouver, BC, Canada). The chicken anti-RP1 antibody ${ }^{19}$ (1:500) was a generous gift of Eric A. Pierce (University of Pennsylvania, Philadelphia, PA). The monoclonal antibody $\mathrm{K}^{26^{20}}$ (1:10) was kindly provided by Joseph C. Besharse (Medical College of Wisconsin, Milwaukee, WI).

\section{Protein Extracts from Tissues, SDS-PAGE, and Western Blot Analysis}

Fresh bovine and porcine eyes, as well as bovine brain stem, heart, and lung were obtained from the local abattoir and stored at $-80^{\circ} \mathrm{C}$. Frozen bovine tissues were homogenized in urea buffer $(8 \mathrm{M}$ urea, $2 \mathrm{M}$ thiourea, 2\% 3-([3-cholamidopropyl]dimethylammonio-2-hydroxy-1propanesulfonate [CHAPS], and $0.1 \mathrm{M}$ dithiothreitol [DTT]), sonicated, and centrifuged at $16,000 \mathrm{~g}$ for 10 minutes at $4^{\circ} \mathrm{C}$. Proteins in the supernatant were added to SDS sample buffer and separated by SDSPAGE on $10 \%$ gels. For Western blot analysis, proteins were electrophoretically transferred onto polyvinylidene difluoride membranes. After the blots were blocked in PBS containing $1 \%$ nonfat dry milk, the membranes were labeled with the primary antibody for 1 hour at room temperature followed by goat anti-rabbit IgG conjugated to horseradish peroxidase (Calbiochem, San Diego, CA). Western blots were developed by chemiluminescence (SuperSignal West Pico Chemiluminescent Substrate; Perbio, Bonn, Germany).

\section{Fractionation of Bovine Retina}

Frozen bovine retina was homogenized in $1 \mathrm{~mL}$ hypotonic buffer (10 $\mathrm{mM}$ Tris- $\mathrm{HCl}[\mathrm{pH} 7.4]$ and $1 \mathrm{mM}$ EDTA containing $1 \mathrm{mM}$ protease inhibitor [Pefabloc SC; Roche Diagnostics, Mannheim, Germany]) at $4^{\circ} \mathrm{C}$. Cell debris and nuclei were removed by low-speed centrifugation $(1000 \mathrm{~g})$. The supernatant was separated into soluble (S2) and membrane (P2) fractions by high-speed centrifugation at 100,000 $\mathrm{g}$ for 30 minutes at $4^{\circ} \mathrm{C}$. The $\mathrm{P} 2$ pellet was resuspended in $0.8 \mathrm{~mL}$ hypotonic buffer, and $0.2 \mathrm{~mL}$ was added to an equal volume of four different extraction buffers (buffer A: $2 \%$ Triton X-100, $0.3 \mathrm{M} \mathrm{NaCl}$; buffer B: $0.4 \%$ SDS, $0.3 \mathrm{M} \mathrm{NaCl}$; buffer C: $0.2 \mathrm{M}$ CHAPS, $2.0 \mathrm{M} \mathrm{NaCl}$; buffer D: 0.4 $\mathrm{M} \mathrm{Na}_{2} \mathrm{CO}_{3}$ [pH 11.0]). After a 30-minute incubation at $4^{\circ} \mathrm{C}$ with gentle stirring, the suspensions were spun down at $100,000 \mathrm{~g}$ for 30 minutes at $4{ }^{\circ} \mathrm{C}$. The resultant pellets $(\mathrm{P} 3)$ were dissolved in $0.4 \mathrm{~mL}$ hypotonic buffer containing $4 \%$ SDS. For SDS-PAGE, $20 \mu \mathrm{L}$ of P3 and supernatant (S3) fractions were mixed with SDS loading buffer and electrophoretically separated on a $10 \%$ gel.

\section{Immunofluorescence Labeling}

Bovine and porcine posterior eye cups were immersion-fixed in $4 \%$ paraformaldehyde in $0.1 \mathrm{M}$ phosphate buffer ( $\mathrm{PB}$; [pH 7.4]) for 2 to 4 hours. After they were rinsed several times in $0.1 \mathrm{M} \mathrm{PB}$, the eyes were 
cryoprotected in $0.1 \mathrm{M} \mathrm{PB}$ containing $18 \%$ sucrose for 12 hours. Bovine and porcine retinas dissected from the eye cups were embedded in optimal cutting temperature (OCT) medium (Tissue-Tek, Miles, Elkhart, IN), fast frozen in liquid nitrogen, and cryosectioned vertically at 8 to $10 \mu \mathrm{m}$. Cryosections were blocked with $0.1 \mathrm{M}$ PB containing $0.3 \%$ Triton X-100 and $10 \%$ goat serum for 30 minutes and labeled for 4 to 12 hours with primary antibodies diluted in $0.1 \mathrm{M} \mathrm{PB}, 0.1 \%$ Triton $\mathrm{X}-100$, and $2.5 \%$ goat serum at room temperature. After they were washed in $0.1 \mathrm{M} \mathrm{PB}$, the sections were incubated with secondary antibodies diluted 1:800 for 1 hour. These included goat anti-rabbit IgG conjugated to Alexa Fluor 488 and goat anti-mouse or anti-chicken IgG conjugated to Alexa Fluor 594 (Molecular Probes, Eugene, OR). Double-labeling studies were performed by simultaneously applying the primary antibodies followed by a mixture of secondary antibodies. Peanut agglutinin-Alexa 594 (Molecular Probes) was applied together with the secondary antibody. Control experiments included the omission of one or both primary antibodies and preabsorption of the diluted affinity-purified polyclonal anti-MPP4 antibody with a 10-fold excess of EP119 peptide $(20 \mu \mathrm{g} / \mathrm{mL})$ for 30 minutes before the staining procedure. Labeled sections were washed in $0.1 \mathrm{M} \mathrm{PB}$ and mounted (Confocal Matrix; MicroTech-Laboratory, Graz, Austria). All labeled specimen were examined under a motorized fluorescence microscope (Axioskop-2 mot plus; Carl Zeiss, Göttingen, Germany). Image processing was achieved on computer (Axiovision software version 3.1 with integrated Z-stack, three-dimensional deconvolution and extended focus modules; Carl Zeiss).

\section{Results}

\section{Isolation of the Full-Length Bovine MPP4 cDNA}

The assembly of overlapping sequences obtained by RT-PCR and RACE experiments on bovine retinal RNA resulted in a 2374-bp full-length bovine MPP4 cDNA. The sequence encompasses an open reading frame of 1917 bp encoding a putative bovine MPP4 protein of 640 amino acids with a predicted molecular mass of $72.9 \mathrm{kDa}$. Compared with human MPP4, the bovine MPP4 sequence contains an additional 9 bp corresponding to an insertion of three amino acids distal to human amino acid position 12. Alignment of the bovine to the human protein sequence identified an overall $94 \%$ similarity and $87 \%$ identity. Regions harboring the conserved protein domains L27N (amino acids [aa] 31-86), L27C (aa 90-143), PDZ (aa 154-238), SH3 (aa 246-313), and GUK (aa 429-622) showed an even higher degree of sequence similarity with up to $98 \%$ identity (e.g., within the PDZ domain).

\section{Characterization of Polyclonal Antibodies to MPP4}

Antibodies recognizing proteins in bovine retina have often been a valuable tool for immunohistochemical and biochemical studies in vision research. ${ }^{20,21}$ To generate a polyclonal antiserum against bovine MPP4, rabbits were immunized with synthetic peptides corresponding to regions of bovine MPP4 that demonstrated no homology to other members of the p55 subfamily of membrane-associated guanylate kinases. Serum samples were tested for specificity by Western blot analysis of glutathione-S-transferase fusion proteins containing the corresponding MPP4 peptides (data not shown). Antisera showing positive responses were further purified by affinity chromatography. The antibody EP119 raised against the N-terminus of bovine MPP4 (aa 2-16) was found to recognize specifically a protein of approximately $70 \mathrm{kDa}$ on Western blot analysis containing bovine retinal extracts (Fig. 1A), which is in agreement with the predicted molecular mass of MPP4. No signals were detected in protein samples extracted from bovine brain stem, lung, and heart, confirming our earlier findings that MPP4 is expressed in the retina. ${ }^{16}$ The specificity of the EP119
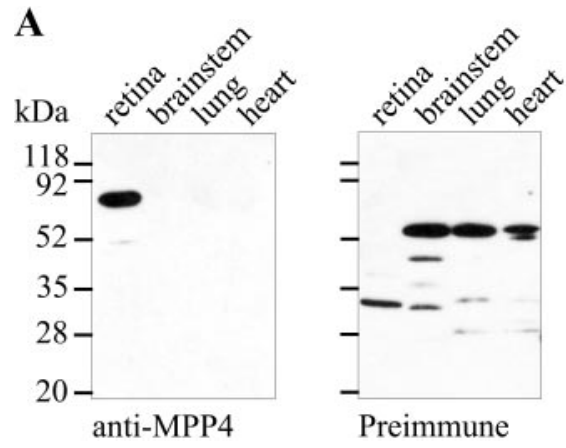

B

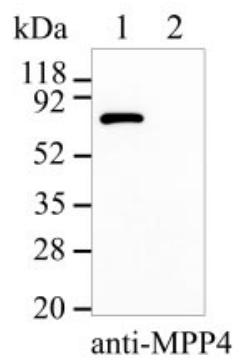

Figure 1. Polyclonal antibodies to the N-terminus of bovine MPP4. (A) Homogenates of bovine tissues (30 $\mu \mathrm{g}$ per lane) were immunoblotted with affinity-purified polyclonal rabbit anti-MPP4 serum or rabbit preimmune serum. (B) Western blot containing lysates of Ebna293 cells transfected with a full-length MPP4 construct (lane 1) or a control plasmid (lane 2) was probed with affinity-purified anti-MPP4 antibody EP119.

antibody was further confirmed by the lack of anti-MPP4 immunoreactivity in the preimmune serum (Fig. 1A). In addition, a protein migrating at $70 \mathrm{kDa}$ was detected in Ebna-293 cell lysates transfected with a full-length MPP4 expression construct but not in cell extracts transfected with a control plasmid (Fig. 1B). From these data, we concluded that the affinitypurified polyclonal anti-MPP4 antibody EP119 specifically recognizes the MPP4 protein.

\section{Distribution of MPP4 in Bovine and Porcine Retina}

The anti-MPP4 antibody EP119 was used to examine the cellular distribution of MPP 4 in cryosections of mammalian retinas by immunofluorescence microscopy. In addition to bovine tissue, the antibody worked well on porcine retina but did not show labeling on mouse or human retinal sections. In both ungulates, a similar pattern of immunostaining in the outer and inner parts of the retina was observed (Fig. 2). This included strong labeling of the junctions between the outer segments (OS) and IS of photoreceptors and the synaptic structures of the OPL, IPL, and fiber layer (FL). Three strata of varicose MPP4-positive terminals were distinguishable in the IPL, wherein the central immunopositive band appeared to be broader in pig than in cow (Figs. 2B, 2E). In porcine but not in bovine retina, cell bodies of presumptive bipolar cells in the outer and central inner nuclear layer (INL), as well as their axonal processes descending into the different strata of the IPL, were moderately labeled (Fig. 2F). Weak MPP4 immunoreactivity was also detected in the IS of cone photoreceptors and the OLM.

Preabsorption with excess MPP4 peptide abolished the labeling of the affinity-purified anti-MPP 4 antibody EP119, confirming the specificity of the MPP4 staining in the retina (data shown for bovine retina in Fig. 2C).

\section{MPP4 in the Connecting Cilia of Cone and Rod Photoreceptors}

To better define the subcellular localization of MPP4 at the link between photoreceptor OS and IS we compared the MPP4 staining on porcine and bovine sections with markers known to be expressed in or adjacent to this ciliary structure. Doublelabeling with the anti-MPP 4 and the anti-rhodopsin antibodies demonstrated no overlap between the rhodopsin expression in the rod outer segments (ROS) and the dotlike arrangement of the MPP4 protein (Fig. 3A). This clearly places the MPP4 proximal to the ROS. MPP4 was also present at the IS-OS 

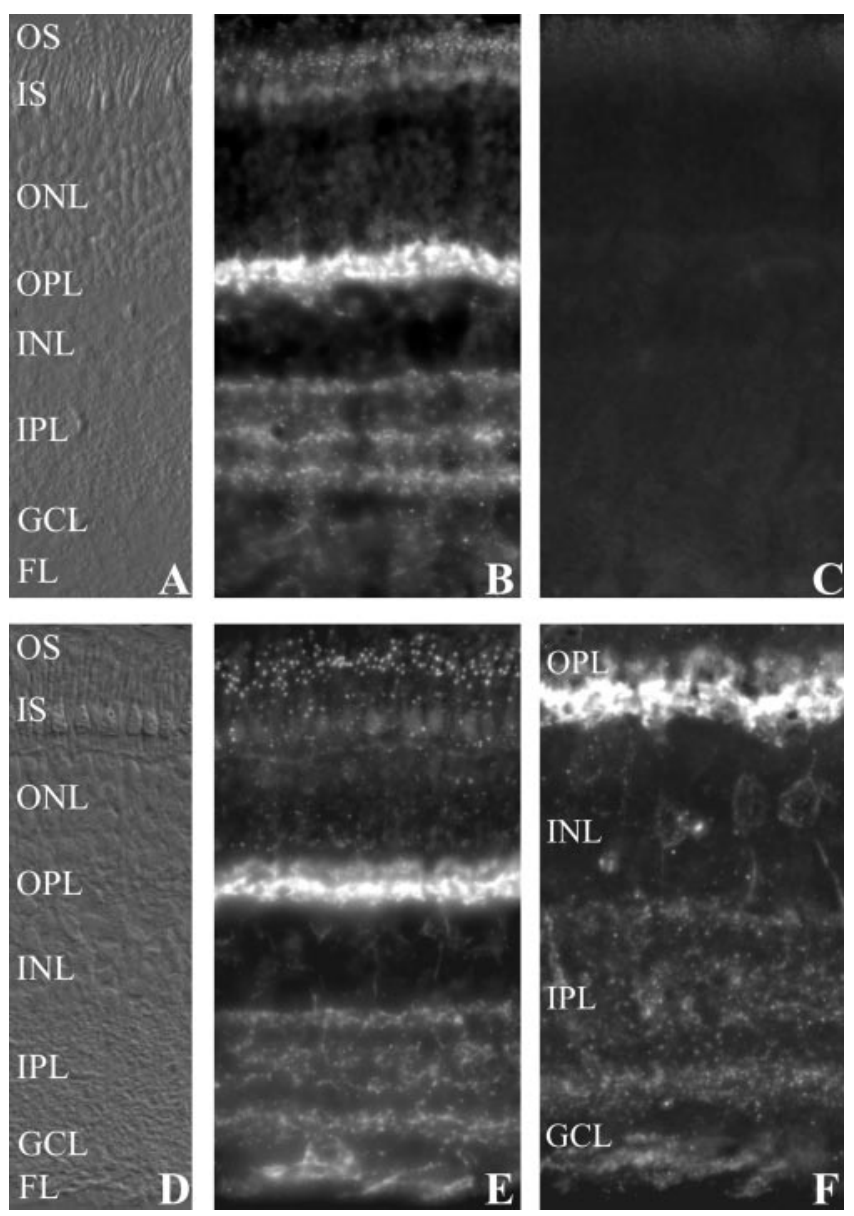

FIGURE 2. Immunofluorescence microscopy of MPP4 in bovine and porcine retinal sections. (A, D) Differential interference contrast (DIC) images showing the various retinal cell layers. (B, E) MPP4 immunolabeling of bovine and porcine retina, respectively. (C) Bovine section labeled with anti-MPP4 antibodies preincubated with an excess of competing MPP4 peptide. (F) MPP4 staining of bipolar cell bodies and descending axons in a porcine section shown at a higher magnification.

junction in cones, as determined by double-labeling with the anti-MPP 4 antibody and peanut agglutinin (PNA), which is known to stain the extracellular matrix that ensheaths cones (Fig. 3B). The RP1 protein has been reported to be distributed along the axonemal microtubules of the connecting cilia in a longitudinal orientation. ${ }^{19}$ More recently, however, the expression of RP1 has been refined to a region distal to the cilium extending into the proximal OS. ${ }^{21}$ Double-labeling using antiMPP4 in conjunction with anti-RP1 antibodies showed the MPP4-stained dots to be immediately proximal to the elongated RP1 structures in rod and cone photoreceptors (Fig. 3C). The RS1 protein is expressed in and secreted by photoreceptor and bipolar cells and has recently been localized to the IS of rod and cone photoreceptors in several mammalian species where it is assumed to be distributed along the surface membranes. ${ }^{18}$ In pig, the MPP4 protein was found to be located distal to the RS1 staining of the rod IS (Fig. 3D). Close inspection of the merged image revealed that the MPP4 puncta were precisely localized in a small space at the distal end of the rod IS that showed no RS1 membrane labeling. In cones, MPP4-stained dots were detected at the distal tips of cone IS, a region that was most intensely labeled by the RS1 antibody (Fig. 3D). Finally, double-labeling with the anti-MPP4 antibody and the monoclonal antibody K26 revealed that the MPP4-immunore- active dots completely coincided with the K26-positive puncta (Fig. 3E). K26 specifically recognizes an unidentified epitope in connecting cilia of bovine photoreceptors. ${ }^{20}$ Taken together, the results of the double-labeling experiments suggest that the MPP4 protein is highly concentrated in the connecting cilia of rod and cone photoreceptors.

\section{Expression of MPP4 in the OPL}

We next sought to characterize further MPP4 immunoreactivity in the OPLs of bovine and porcine retinal sections. In the OPL of the bovine retina, the anti-MPP 4 antibody was observed to label intensely the membranes of cone pedicles and rod spherules. This was verified by double-labeling with the antiMPP4 antibody in combination with antibodies to VGLUT1, which is a vesicular glutamate receptor expressed in the synaptic vesicles of photoreceptor terminals. ${ }^{22}$ MPP4-positive presynaptic membranes ensheathed the VGLUT1 immunolabeling in the photoreceptor cells (Fig. 4A). In addition, MPP4 colocalized with membrane-associated PSD-95 at the basal membranes of the photoreceptor terminals (Fig. 4B). On porcine sections, the MPP4 staining of the cone and rod terminals appeared to be weaker but essentially coincided with the PSD-95 immunolabeling (Fig. 4C). The available VGLUT1 antibody did not label the porcine retina.

In bovine, but more prominent in porcine retina, additional MPP4-immunopositive structures were observed in the OPL. This non-photoreceptor staining was most intense below presumptive cone pedicles along the outer margin of the OPL. As a first step in analyzing the nature of the postsynaptic elements in the OPL we labeled retinal sections with an antibody directed against calretinin, a calcium-binding protein that is expressed in horizontal cells of mammals. ${ }^{23,24}$ In porcine and bovine retinas, the calretinin antibody labeled the cell bodies of horizontal cells in the outer part of the INL. Stained processes and punctate structures represent invaginating horizontal cell tips that contact the photoreceptor terminals (Figs. 4D, 4E). On double-labeling, the MPP4 immunoreactivity appeared to be different from the calretinin-labeled horizontal processes (Figs. 4D, 4E). An antibody against ROB that has been found to label rod bipolar cells and a subset of ON cone bipolar cells in vertebrates $^{25,26}$ was applied in conjunction with the anti-MPP4 antibody. The merged image showed an overlap between the ROB-immunoreactive dendrites and the MPP4-positive structures but also revealed several diffuse fibers in the inner OPL that were labeled only by the anti-MPP4 antibody (shown for porcine retina in Fig. 4F). From this, we conclude that the anti-MPP4 antibody EP119 produces specific labeling of presynaptic membranes in rod and cone photoreceptors and stains structures in the inner part of the OPL that presumably include the membranes of bipolar cell dendrites.

\section{Expression of MPP4 in the Inner Retina}

Two bipolar cell markers were used to study more precisely the subset of neurons labeled by the anti-MPP 4 antibody in pig (Figs. 5A-D). The antibody directed against PKC $\alpha$ stained the rod bipolar cell perikarya located in the outer part of the INL with their axons terminating close to the IPL-GCL border. Double-labeling with the anti-MPP4 antibody showed no immunoreactivity of MPP4 in rod bipolar cells and is consistent with the absence of MPP4-positive terminals in the outermost sublamina of the IPL (Figs. 5A, 5B). The ROB antibody labeled rod and many cone bipolar cell bodies in the outer and central INL and produced a homogenous immunostaining in the IPL with two more intensely labeled bands at the more distal border and the center of the IPL. When this section was stained with the anti-MPP 4 antibody, only a few ROB-positive bipolar cells expressed MPP4 (Figs. 5C, 5D), suggesting that MPP 4 is 

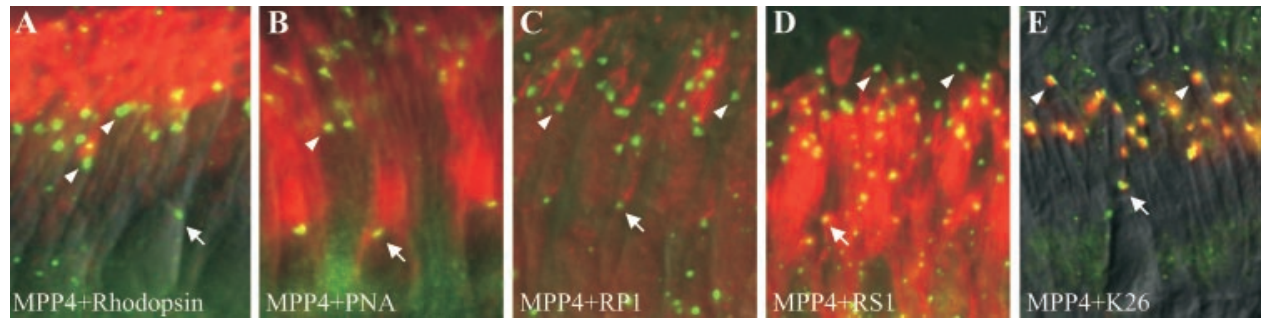

FigURE 3. Localization of MPP4 to the connecting cilia of photoreceptors. Immunofluorescence micrographs of porcine (A- D) or bovine (E) retina double-labeled with the anti-MPP4 antibody (green); the (A) anti-rhodopsin, (B) Alexa 594-conjugated peanut agglutinin, (C) anti-RP1, and (D) anti-RS1 antibodies; and the (E) K26 monoclonal antibody (all red) were merged with differential interference contrast images of the respective retinal sections. Yellow indicates colocalization. Arrows and arrowheads: examples of labeled cone and rod connecting cilia, respectively.

present in a different subgroup of cone bipolar cells. Doublelabeling with antibodies to glutamine synthetase (a Müller cell marker) demonstrated that MPP4 is not expressed in the cell bodies of this cell type (data not shown).

MPP4 immunofluorescence showed a trilaminar pattern of synaptic structures in the IPL. Axonal processes of MPP4positive porcine cone bipolar cells were observed to ramify in these layers, indicating that most of the MPP4 labeling in the IPL represented bipolar cell terminals. This is in agreement with the lack of MPP 4 immunoreactivity in calretinin-positive amacrine cells and their dendrites in the IPL, as shown by double-labeling of a porcine section (Figs. 5E, 5F). Similar results were obtained in bovine retina (data not shown).

\section{Subcellular Distribution of MPP4}

Immunofluorescence staining of Ebna-293 cells expressing bovine MPP4 showed a predominant localization of MPP 4 to the plasma membrane (Fig. 6A). To investigate a plasma membrane association of MPP4 in native tissue, subcellular fractionation of bovine retina was performed that revealed an enrichment of MPP4 in the crude membrane (P2) fraction. In extraction
FIGURE 4. Immunofluorescence microscopic observations of MPP4 expression in the OPL. Bovine retinal sections simultaneously stained with the anti-MPP4 antibody (red) and antibodies directed to (A) VGLUT1, (B) PSD-95, and (D) calretinin (all green). Porcine retinal sections double-labeled with the anti-MPP4 (red) antibody and the (C) anti-PSD-95, (E) anti-calretinin, and (F) anti-ROB antibodies (all green). Merged images are shown in the right column. Yellow indicates colocalization. Arrows: examples of labeled cone pedicles; $a r$ rowheads: examples of labeled rod spherules.
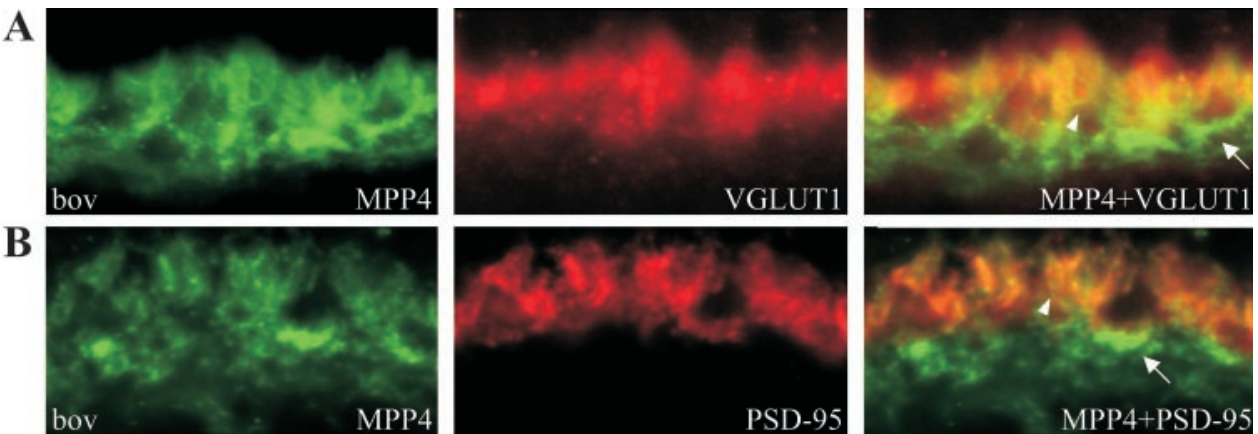

C
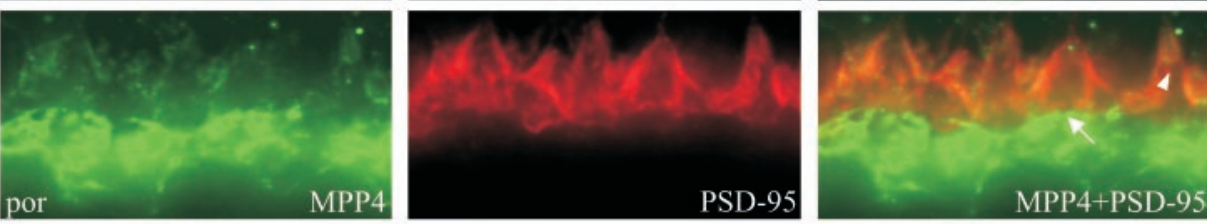

D
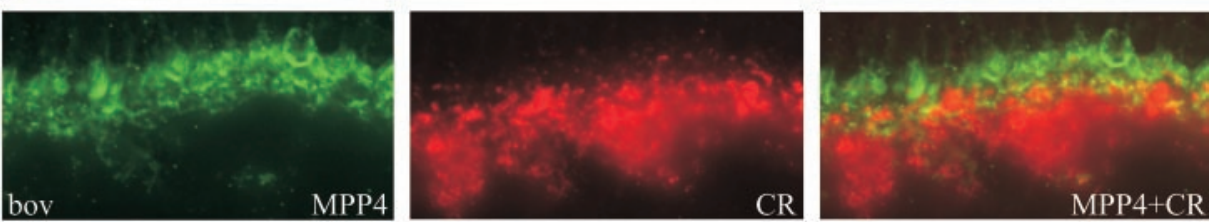

E
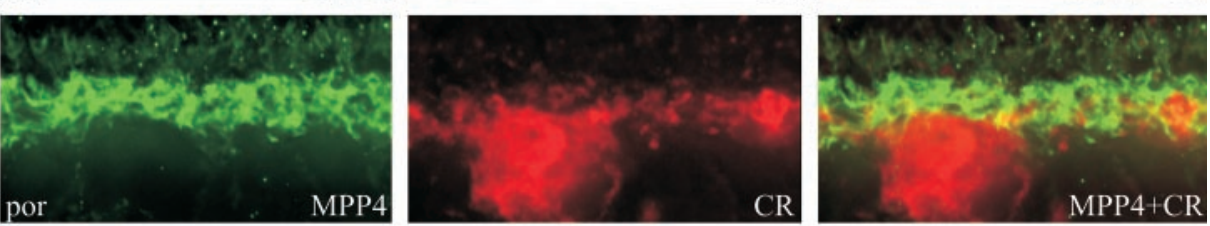

F
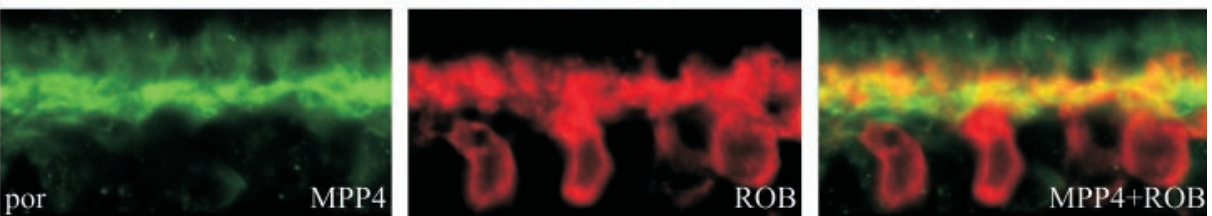

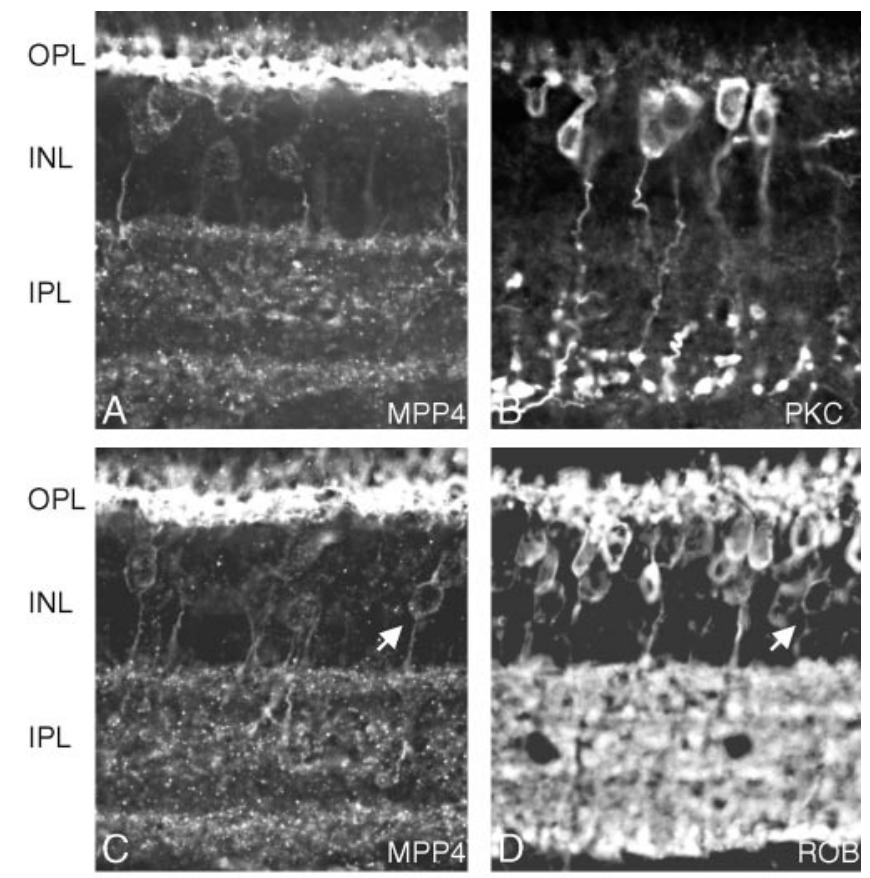

IPL
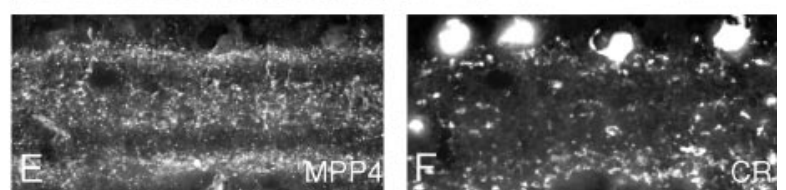

FIGURE 5. Immunofluorescence microscopy of MPP4 in the porcine inner retina. Sections of porcine retina were double-labeled with the anti-MPP4 antibody (A, C, E) and the anti-PKC $\alpha$ (B), anti-ROB (D), and anti-calretinin (F) antibodies. (C, D, arrow) Bipolar cell stained with the anti-MPP4 and anti-ROB antibodies.

experiments of P2 membranes with several agents, MPP4 was largely resistant to solubilization with the nonionic detergent Triton X-100 (Fig. 6B). In contrast, the ionic detergent SDS completely solubilized MPP4. A mixture of the zwitter ionic detergent CHAPS and high salt and alkaline conditions also solubilized significant amounts of MPP4 (Fig. 6B), indicating that MPP4 is not an integral membrane protein but is associated with membrane-bound cytoskeletal elements in the retinal tissue.

\section{Discussion}

The knowledge about the cellular localization of a protein in the highly specialized multilayered retina often provides im- portant hints as to its function in vivo. In the present study, the retina-specific MPP4 protein was found to be localized to several distinct spatial and functional compartments in the outer and inner parts of the mammalian retina. In both rod and cone photoreceptors of bovine and porcine eyes, MPP4 was specifically expressed in the connecting cilia and the synaptic membranes of cone pedicles and rod spherules, domains within the sensory neurons that have unique structures and functions. MPP4 was also found postsynaptic to the photoreceptor ribbon synapses in the OPL and in three defined laminar structures in the IPL. Although the presence and functional importance of MAGUK proteins at cell junctions including synapses is a common feature among the various members of this protein family, MPP4 is the first MAGUK protein that has been localized to ciliary structures.

The connecting cilium is a narrow bridge linking the lightperceiving OS with the IS that contains the protein synthesis machinery. It is homologous to the transitional zone of a motile cilium $^{27}$ and encompasses the microtubule-based axoneme that arises from the basal body at the tip of the IS and runs through the entire length of the cilium into the proximal OS. Two major roles in photoreceptor function have been assigned to the connecting cilium: the active transport of newly synthesized molecules (e.g., phototransduction proteins, disc lipids) from the IS to the basis of the $\operatorname{OS}^{28,29}$ and disc membrane morphogenesis. ${ }^{30}$ The latter takes place within small actincontaining domains at the base of the OS where membrane proteins are incorporated into evaginating plasma membranes that form the nascent disks. ${ }^{31}$

On the basis of what is known about other MAGUK family members, it is intriguing to speculate that MPP4 participates in ciliary function by providing a link between components of the cytoskeleton and the plasma membrane or cell surface molecules, thereby contributing to the stability of the cilium and the maintenance of distinct membrane domains. MAGUK protein p55/MPP1, for example, interacts with glycophorin $\mathrm{C}$ and protein 4.1 to form a ternary complex in the erythrocyte membrane that regulates the stability and mechanical properties of the erythrocyte plasma membrane. ${ }^{32}$ CASK also interacts with protein 4.1 and nucleates the assembly of actin/ spectrin filaments. ${ }^{33} \mathrm{~A}$ different cytoskeletal link has been demonstrated for PSD-93 that interacts with microtubule-associated protein 1A (MAP1A) through its guanylate kinase domain. ${ }^{34}$ Future studies to identify interactions between MPP4 and other ciliary proteins are likely to elucidate further the role of MPP4 in the connecting cilium.

In recent years, several proteins have been identified as part of the ciliary apparatus of photoreceptors including components of the microtubule and actin cytoskeleton ${ }^{35,31}$; nonconventional cytoskeletal proteins (centrin and myocilin) ${ }^{36,37}$; KIF3A, a kinesin motor protein ${ }^{38}$; unconventional myosin
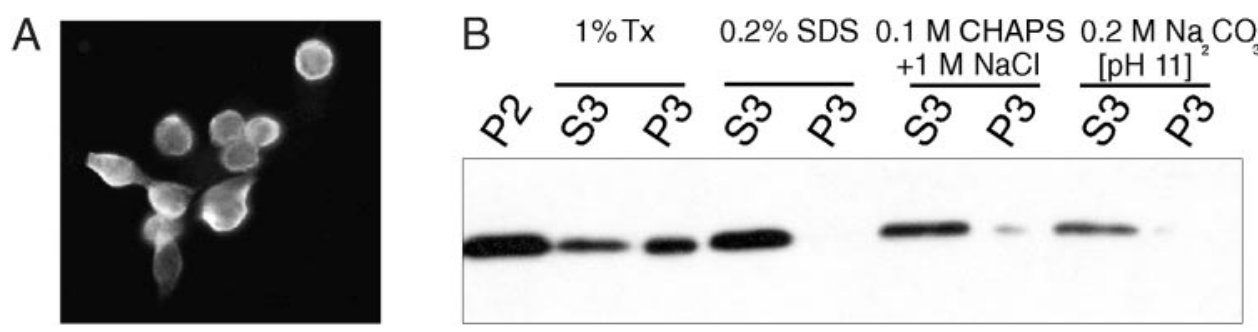

FIGURE 6. MPP4 is associated with the plasma membrane and the cytoskeleton. (A) Distribution of transiently expressed MPP4 in Ebna-293 cells. (B) Extraction of the bovine crude retinal membrane fraction (P2) with various detergents. Lanes marked S3 contain the detergent-soluble fractions and lanes marked P3 the insoluble fractions (20 $\mu \mathrm{g}$ per lane). Proteins were Western blotted and probed with affinity-purified anti-MPP4 antibodies. 
$\mathrm{VIIa}^{39}$; retinitis pigmentosa GTPase regulator (RPGR) and the RPGR-interacting protein (RPGRIP), which are believed to be involved in disk morphogenesis ${ }^{40}$; and the RP1 protein, a putative member of the protein transport machinery. ${ }^{19}$ The latter four proteins are products of genes that have been implicated in different forms of retinal degeneration-namely, retinitis pigmentosa (RPGR and RP1), ${ }^{41,42}$ Leber congenital amaurosis (RPGRIP), ${ }^{43}$ and Usher syndrome $1 \mathrm{~B} .{ }^{44}$ The mutational analysis of the MPP 4 gene in 300 patients with retinitis pigmentosa (RP) and a family with autosomal recessive RP genetically linked to the MPP4 locus on the long arm of chromosome 2 at bands 2q31-33 (RP26) revealed no pathologic mutations. ${ }^{45} \mathrm{RP}$, however, is a genetically and clinically heterogenous group of retinal degenerations and an implication of MPP4 in this or other forms of retinopathy is still conceivable.

Unlike PSD-95, PSD-93, SAP102, and SAP97, the expression of which is restricted to either the pre- or postsynaptic site of the photoreceptor synapse, MPP 4 seems to be present on both sides of the synaptic cleft. At the membrane of the photoreceptor terminal, MPP4 appears to colocalize with PSD-95. It has been demonstrated that PSD-95 binds to other MAGUK family members and this interaction is mediated by heterodimerization of the respective L27 domains. ${ }^{46}$ MPP4 possesses two tandem L27 motifs in its N-terminal half that makes it an attractive binding partner for PSD-95 in the photoreceptor synapse.

The double-labeling experiments performed in this study suggest that the MPP4 immunoreactivity in the postsynaptic OPL and in the IPL is mainly due to MPP 4 expression in bipolar cells dendrites and their axonal terminals, respectively. This is consistent with the observation that a specific subset of cone bipolar cells was labeled by the anti-MPP 4 antibody on porcine sections. That bovine bipolar cells do not show any detectable MPP4 staining can be attributed to the well-known speciesspecific differences in bipolar cell markers. ${ }^{47}$ To determine precisely the origin of the postsynaptic structures in the OPL and the three labeled strata in the IPL, more detailed investigations with additional markers and higher resolution microscopy are necessary.

A close relative of MPP4, the zebrafish orthologue of MPP5 has been localized apical to the OLM in zebrafish retina, ${ }^{15}$ a region that is preferentially labeled by antibodies to Drosophila Crumbs and mouse Crb1, putative binding partners of orthologous MPP5 proteins. ${ }^{13}$ The photoreceptor IS and OLM structures of bovine and porcine retina were faintly positive for MPP4. However, a concentration of MPP4 protein in or adjacent to the OLM was not detected with the antibody used in this study. This suggests that MPP 4 and -5 are part of different molecular complexes in the mammalian retina.

The multiple subcellular locations of MPP4 in bovine and porcine retinas implies that MPP4 contributes to several functional aspects of the mammalian retina. Preliminary biochemical data showed that MPP4 is largely resistant to detergent solubilization of retinal membranes which is consistent with MPP4's being associated with elements of the ciliary and synaptic cytoskeleton underlying the plasma membranes. Additional functional analyses are needed to define more closely the components of the putative protein complexes organized by MPP4. This, in turn may provide insight into its function and may possibly clarify its potential role in retinal diseases.

\section{References}

1. Dimitratos SD, Woods DF, Stathakis DG, et al. Signaling pathways are focused at specialized regions of the plasma membrane by scaffolding proteins of the MAGUK family. Bioessays. 1999;21: 912-921.
2. Doerks T, Bork P, Kamberov E, et al. L27, a novel heterodimerization domain in receptor targeting proteins Lin-2 and Lin-7. Trends Biochem Sci. 2000;25:317-318.

3. Bertin J, Wang L, Guo Y, et al. CARD11 and CARD14 are novel caspase recruitment domain (CARD)/membrane-associated guanylate kinase (MAGUK) family members that interact with BCL10 and activate NF-kappa B. J Biol Chem. 2001;276:11877-11882.

4. Koulen P, Fletcher EL, Craven SE, et al. Immunocytochemical localization of the postsynaptic density protein PSD-95 in the mammalian retina. J Neurosci. 1998;18:10136-10149.

5. Koulen P. Localization of synapse-associated proteins during postnatal development of the rat retina. EurJ Neurosci. 1999;11:20072018.

6. Koulen P, Garner CC, Wässle H. Immunocytochemical localization of the synapse-associated protein SAP102 in the rat retina. J Comp Neurol. 1998;397:326-336.

7. Roh MH, Makarova O, Liu CJ, et al. The Maguk protein, Pals1, functions as an adapter, linking mammalian homologues of Crumbs and Discs Lost. J Cell Biol. 2002;157:161-172.

8. den Hollander AI, ten Brink JB, de Kok YJ, et al. Mutations in a human homologue of Drosophila crumbs cause retinitis pigmentosa (RP12). Nat Genet. 1999;23:217-221.

9. Lotery AJ, Jacobson SG, Fishman GA, et al. Mutations in the CRB1 gene cause Leber congenital amaurosis. Arch Ophthalmol. 2001; 119:415-420.

10. Bhat MA, Izaddoost $S$, Lu Y, et al. Discs Lost, a novel multi-PDZ domain protein, establishes and maintains epithelial polarity. Cell. 1999;96:833-845.

11. Hong Y, Stronach B, Perrimon N, et al. Drosophila Stardust interacts with Crumbs to control polarity of epithelia but not neuroblasts. Nature. 2001;414:634-638.

12. Bachmann A, Schneider M, Theilenberg E, et al. Drosophila Stardust is a partner of Crumbs in the control of epithelial cell polarity. Nature. 2001;414:638-643.

13. Pellikka M, Tanentzapf G, Pinto M, et al. Crumbs, the Drosophila homologue of human CRB1/RP12, is essential for photoreceptor morphogenesis. Nature. 2002;416:143-149.

14. Izaddoost S, Nam SC, Bhat MA, et al. Drosophila Crumbs is a positional cue in photoreceptor adherens junctions and rhabdomeres. Nature. 2002;416:178-183.

15. Wei X, Malicki J. nagie oko, encoding a MAGUK-family protein, is essential for cellular patterning of the retina. Nat Genet. 2002;31: $150-157$.

16. Stöhr H, Weber BHF. Cloning and characterization of the human retina-specific gene MPP4, a novel member of the p55 subfamily of MAGUK proteins. Genomics. 2001;74:377-384.

17. MacKenzie D, Arendt A, Hargrave P, et al. Localization of binding sites for carboxyl terminal specific anti-rhodopsin monoclonal antibodies using synthetic peptides. Biochemistry. 1984;23:65446549.

18. Molday LL, Hicks D, Sauer CG, et al. Expression of X-linked retinoschisis protein RS1 in photoreceptor and bipolar cells. Invest Ophthalmol Vis Sci. 2001;42:816-825.

19. Liu Q, Zhou J, Daiger SP, et al. Identification and subcellular localization of the RP1 protein in human and mouse photoreceptors. Invest Ophthalmol Vis Sci. 2002;43:22-32.

20. Horst CJ, Johnson LV, Besharse JC. Transmembrane assemblage of the photoreceptor connecting cilium and motile cilium transition zone contain a common immunologic epitope. Cell Motil Cytoskeleton. 1990;17:329-344.

21. Hong DH, Pawlyk B, Sokolov M, et al. RPGR isoforms in photoreceptor connecting cilia and the transitional zone of motile cilia. Invest Ophthalmol Vis Sci. 2003;44:2413-2421.

22. Johnson J, Tian N, Caywood MS, et al. Vesicular neurotransmitter transporter expression in developing postnatal rodent retina: GABA and glycine precede glutamate. J Neurosci. 2003;23:518529.

23. Pasteels B, Rogers J, Blachier F, et al. Calbindin and calretinin localization in retina from different species. Vis Neurosci. 1990;5: 1-16.

24. Haverkamp S, Wassle H. Immunocytochemical analysis of the mouse retina. J Comp Neurol. 2000;424:1-23. 
25. Onoda N, Fujita SC. A monoclonal antibody specific for a subpopulation of retinal bipolar cells in the frog and other vertebrates. Brain Res. 1987; 416:359-363.

26. Grunert U, Martin PR, Wassle H. Immunocytochemical analysis of bipolar cells in the macaque monkey retina. J Comp Neurol. 1994;348:607-627.

27. Rohlich P. The sensory cilium of retinal rods is analogous to the transitional zone of motile cilia. Cell Tissue Res. 1975;161:421430 .

28. Wolfrum U, Schmitt A. Rhodopsin transport in the membrane of the connecting cilium of mammalian photoreceptor cells. Cell Motil Cytoskeleton. 2000;46:95-107.

29. Marszalek JR, Liu X, Roberts EA, et al. Genetic evidence for selective transport of opsin and arrestin by kinesin-II in mammalian photoreceptors. Cell. 2000;102:175-187.

30. Williams DS, Hallett MA, Arikawa K. Association of myosin with the connecting cilium of rod photoreceptors. J Cell Sci. 1992;103: 183-190.

31. Chaitin MH, Schneider BG, Hall MO, et al. Actin in the photoreceptor connecting cilium: immunocytochemical localization to the site of outer segment disk formation. J Cell Biol. 1984;99:239-247.

32. Chishti AH. Function of p55 and its nonerythroid homologues. Curr Opin Hematol. 1998;5:116-121.

33. Biederer T, Sudhof TC. CASK and protein 4.1 support F-actin nucleation on neurexins. J Biol Chem. 2001;276:47869-47876.

34. Brenman JE, Topinka JR, Cooper EC, et al. Localization of postsynaptic density-93 to dendritic microtubules and interaction with microtubule-associated protein 1A. J Neurosci. 1998;18:8805-8813.

35. Sale WS, Besharse JC, Piperno G. Distribution of acetylated alphatubulin in retina and in vitro-assembled microtubules. Cell Motil Cytoskeleton. 1988;9:243-253.

36. Wolfrum U. Centrin in the photoreceptor cells of mammalian retinae. Cell Motil Cytoskeleton. 1995;32:55-64.

37. Kubota R, Noda S, Wang Y, et al. A novel myosin-like protein (myocilin) expressed in the connecting cilium of the photoreceptor: molecular cloning, tissue expression, and chromosomal mapping. Genomics. 1997;41:360-369.

38. Whitehead JL, Wang SY, Bost-Usinger L, et al. Photoreceptor localization of the KIF3A and KIF3B subunits of the heterotrimeric microtubule motor kinesin II in vertebrate retina. Exp Eye Res. 1999;69:491-503.

39. Liu X, Vansant G, Udovichenko IP, et al. Myosin VIIa, the product of the Usher 1B syndrome gene, is concentrated in the connecting cilia of photoreceptor cells. Cell Motil Cytoskeleton. 1997;37:240 252.

40. Zhao Y, Hong DH, Pawlyk B, et al. The retinitis pigmentosa GTPase regulator (RPGR)-interacting protein: subserving RPGR function and participating in disk morphogenesis. Proc Natl Acad Sci USA. 2003;100:3965-3970.

41. Meindl A, Dry K, Herrmann K, et al. A gene (RPGR) with homology to the RCC1 guanine nucleotide exchange factor is mutated in X-linked retinitis pigmentosa (RP3). Nat Genet. 1996;13:35-42.

42. Sullivan LS, Heckenlively JR, Bowne SJ, et al. Mutations in a novel retina-specific gene cause autosomal dominant retinitis pigmentosa. Nat Genet. 1999;22:255-259.

43. Dryja T P, Adams SM, Grimsby JL, et al. Null RPGRIP1 alleles in patients with Leber congenital amaurosis. Am J Hum Genet. 2001; 68:1295-1298.

44. Weil D, Blanchard S, Kaplan J, et al. Defective myosin VIIA gene responsible for Usher syndrome type 1B. Nature. 1995;374:60 61

45. Conte I, Lestingi M, den Hollander A, et al. Characterization of MPP4, a gene highly expressed in photoreceptor cells, and mutation analysis in retinitis pigmentosa. Gene. 2002;297:33-38.

46. Chetkovich DM, Bunn RC, Kuo SH, et al. Postsynaptic targeting of alternative postsynaptic density-95 isoforms by distinct mechanisms. J Neurosci. 2002;22:6415-6425.

47. Haverkamp S, Ghosh KK, Hirano AA, et al. Immunocytochemical description of five bipolar cell types of the mouse retina. J Comp Neurol. 2003;455:463-476. 